\title{
Functional characterization of a fatty acyl-CoA- binding protein (ACBP) from the apicomplexan Cryptosporidium parvum
}

\author{
Bin Zeng, Xiaomin Cai and Guan Zhu \\ Department of Veterinary Pathobiology, College of Veterinary Medicine \& Biomedical Sciences, \\ Texas A\&M University, 4467 TAMU, College Station, TX 77483-4467, USA
}

Correspondence

Guan Zhu

Gzhu@cvm.tamu.edu

Received 22 February 2006

Revised 15 April 2006

Accepted 24 April 2006

\begin{abstract}
In this paper, the identification and functional analysis of a fatty acyl-CoA-binding protein (ACBP) gene from the opportunistic protist Cryptosporidium parvum are described. The CpACBP1 gene encodes a protein of 268 aa that is three times larger than typical ACBPs (i.e. $\sim 90$ aa) of humans and animals. Sequence analysis indicated that the CpACBP1 protein consists of an $\mathrm{N}$-terminal ACBP domain ( $90 \mathrm{aa}$ ) and a C-terminal ankyrin repeat sequence $(\sim 170 \mathrm{aa})$. The entire $C p A C B P 1$ ORF was engineered into a maltose-binding protein fusion system and expressed as a recombinant protein for functional analysis. Acyl-CoA-binding assays clearly revealed that the preferred binding substrate for CpACBP1 is palmitoyl-CoA. RT-PCR, Western blotting and immunolabelling analyses clearly showed that the $C p A C B P 1$ gene is mainly expressed during the intracellular developmental stages and that the level increases during parasite development.

Immunofluorescence microscopy showed that CpACBP1 is associated with the parasitophorous vacuole membrane (PVM), which implies that this protein may be involved in lipid remodelling in the $\mathrm{PVM}$, or in the transport of fatty acids across the membrane.
\end{abstract}

\section{INTRODUCTION}

Cryptosporidium parvum is a globally important parasitic protist that infects both humans and animals (Chappell \& Okhuysen, 2002; Thompson et al., 2005; Tzipori \& Widmer, 2000). C. parvum belongs to the phylum Apicomplexa that contains many important human and animal parasites (e.g. Plasmodium, Babesia, Toxoplasma and Eimeria) (Zhu et al., 2000a). This group of parasites shares some common biological features characteristic to the phylum. For example, all apicomplexans possess similar complex lifecycle stages, including oocyst formation, sporulation, merogony and gametogenesis. However, recent advances in biochemistry and genome sequencing have revealed that a number of metabolic pathways, such as fatty acid biosynthesis, are highly diverse within the Apicomplexa (Abrahamsen et al., 2004; Zhu et al., 2000a). Apicomplexans may possess apicoplast-specific Type II fatty acid synthases (e.g. Plasmodium falciparum), Type I fatty acid synthase (e.g. C. parvum) or both (e.g. Toxoplasma gondii) (Roos et al., 2002; Zhu, 2004; Zhu et al., 2000b, 2002, 2004). Although fatty acids are one of the major components in all cells, free

Abbreviations: ACBP, acyl-CoA-binding protein; PPT, phosphopantetheinyl transferase; GPI, glycosylphosphatidylinositol; MBP, maltosebinding protein; PVM, parasitophorous vacuole membrane; SFP, surfactin production element; TMP, total membrane proteins.

The GenBank/EMBL/DDBJ accession number for the sequence reported in this paper is DQ406676 fatty acids cannot enter any metabolic pathways unless they are activated by thioesterification with coenzyme A (CoA), to form an acyl-CoA ester, or with acyl carrier protein. Fatty acyl-CoA can immediately enter subsequent metabolic pathways or may be stored/transported by a family of acyl-CoA-binding proteins (ACBPs).

ACBPs are a group of highly conserved proteins and have been found in animals, plants, protists and a number of pathogenic bacteria (Burton et al., 2005). They are typically small, cytosolic molecules of $\sim 10 \mathrm{kDa}$. However, a number of larger ACBPs (e.g. $>55 \mathrm{kDa}$ ) have also been identified in both animals and plants. Mammals possess multiple ACBPs that are differentially expressed in various tissues (e.g. T-ACBP in testis, L-ACBP in liver and I-ACBP in intestine) (Schroeder et al., 1998). In Trypanosoma brucei, an ACBP was found to be involved in the synthesis of the glycosylphosphatidylinositol (GPI) anchor in variant surface glycoproteins (Milne \& Ferguson, 2000; Milne et al., 2001). Although ACBPs are capable of binding mediumto long-chain fatty acyl-CoA esters, they may vary in their substrate preference and binding affinities. For example, the highest affinities of ACBPs from bovines (liver) and trypanosomes (or P. falciparum) are C18 stearoyl- and C14 lauroyl-CoA, respectively (Milne \& Ferguson, 2000; van Aalten et al., 2001).

By data-mining the recently completed C. parvum genome sequence, we have identified a unique long-type ACBP 
(CpACBP1) that contains an N-terminal ACBP domain and a C-terminal ankyrin-repeat sequence, which is structurally similar to the membrane-associated ACBP1 and ACBP2 in Arabidopsis thaliana. In the present study, we have expressed CpACBP1 protein as a fusion protein and characterized its primary biochemical features. We have also found that the $C P A C B P 1$ gene is differentially expressed during the parasite life cycle, and the protein it encodes is chiefly located on the parasitophorous vacuole membrane (PVM), suggesting that this protein may be involved in the formation of PVM and/ or the uptake of fatty acids by the parasite.

\section{METHODS}

Identification of ACBP homologues from $C$. parvum and other apicomplexans. $C P A C B P 1$ was identified from the $C$. parvum genome as an intronless gene by a homology search using characterized animal and plant ACBPs as query sequences. Similar searches were also performed to identify ACBP homologues from the Plasmodium falciparum and Toxoplasma gondii genome databases (www.PlasmoDB.org and www.ToxoDB.org, respectively) for comparison. Domains in the apicomplexan ACBP homologues were identified by searching the Conserved Domain Database at the National Center for Biotechnology Information (NCBI, www.ncbi. nlm.nih.gov) and by comparison with other characterized proteins in the GenBank database.

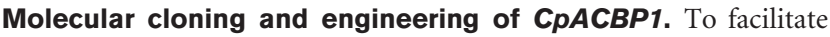
biochemical analysis, we cloned $C P A C B P 1$ and expressed CpACBP1 as a maltose-binding protein (MBP) fusion as described below. Briefly, the entire ORF of $C P A C B P 1$ was amplified from C. parvum (Iowa strain) genomic DNA with the following primer pair: $5^{\prime}$ ATGACTGATATCTTATCCACGAAC-3' and $5^{\prime}$-atggatccTTAACTGCTTTCGAGAATTCTT-3' (lower case letters represent an added $B a m H I$ restriction site). A high-fidelity Pfu DNA polymerase (Stratagene) was used to minimize potential errors introduced by amplification. The PCR product was digested with BamHI to produce a cohesive $3^{\prime}$ end, but retained the blunt $5^{\prime}$ end to facilitate unidirectional cloning. The $5^{\prime}$ ends were phosphorylated by treating the amplicons with T4 polynucleotide kinase. After agarose gel electrophoresis, DNA fragments of the expected size were purified using a MinElute gel extraction kit (Qiagen) and ligated into an $X m n I$ and BamHI double-digested pMAL-c2x vector (New England Biolabs) with T4 DNA ligase. The ligated plasmids were transformed into Escherichia coli TOPO-10 (Invitrogen). The resulting colonies were first screened using a sense-stranded primer located upstream from the insert in the vector and the CPACBP1 antisense strand primer. Plasmids were isolated from PCR-positive colonies for sequencing to confirm their identity and the sequence of the inserts. The resulting construct and encoded fusion protein were named pMAL-c2xCpACBP1 and MBP-CpACBP1, respectively.

Similarly, we also constructed an MBP fusion containing only the ACBP domain. Because of the presence of two EcoRI restriction sites flanking the ankyrin domain (i.e. nt 298-788), we removed the entire C-terminal ankyrin repeats by digesting pMAL-c2x-CpACBP1 with EcoRI followed by agarose gel separation. The remaining plasmid fragment was religated back, resulting in a construct (pMAL-c2xCpACBP1-S) encoding only the N-terminal 100 aa plus 7 extra aa derived from the remaining nucleotides at the $3^{\prime}$ end of the insert and the multiple cloning site of the vector (MBP-CpACBP1-S). We predict that the extra 7 aa (RKQLRIL) will not alter the function of the ACBP domain as they do not resemble any known functional sequences in the databases.
For each construct, a plasmid containing the correct insert was transformed into E. coli Rosetta cells (Novagen) for protein expression. Briefly, a single clone of the E. coli transformants was inoculated into $10 \mathrm{ml} \mathrm{LB}$ broth containing $100 \mu \mathrm{g}$ ampicillin $\mathrm{ml}^{-1}$ and $34 \mu \mathrm{g}$ chloramphenicol ml $\mathrm{m}^{-1}$ and grown overnight at $37^{\circ} \mathrm{C}$. On the second day, the bacterial suspension was transferred into 11 fresh medium containing the two antibiotics and grown at $30{ }^{\circ} \mathrm{C}$ until the $\mathrm{OD}_{495}$ reached $0 \cdot 3-0 \cdot 5$. After adding IPTG to the broth $\left(100 \mu \mathrm{g} \mathrm{ml}^{-1}\right.$ final concn), bacteria were further incubated for $4 \mathrm{~h}$ at $30^{\circ} \mathrm{C}$ and collected by centrifugation for $10 \mathrm{~min}$ at $8000 \mathrm{~g}$. Bacterial pellets were resuspended in $50 \mathrm{ml}$ column buffer $(20 \mathrm{mM}$ Tris/ $\mathrm{HCl}, \mathrm{pH} 7 \cdot 5$, $20 \mathrm{mM} \mathrm{NaCl}, 2 \mathrm{mM}$ EDTA) containing a protease inhibitor cocktail (Sigma-Aldrich), disrupted by sonication and centrifuged $(8000 \mathrm{~g}$, $10 \mathrm{~min}$ ) to remove cell debris. Supernatants were applied to an amylose-resin column (New England Biolabs), washed with column buffer ( $>10 \times$ bed vol.) and the MBP-CpACBP1 fusion proteins were eluted from the column with elution buffer $(10 \mathrm{mM}$ maltose, $20 \mathrm{mM}$ Tris/ $\mathrm{HCl}, \mathrm{pH} 7 \cdot 5,20 \mathrm{mM} \mathrm{NaCl}, 2 \mathrm{mM}$ EDTA) according to the manufacturer's protocol. The size and purity of the recombinant proteins were analysed by SDS-PAGE. Protein concentrations were determined by the Bradford (1976) method using a commercial kit and bovine serum albumin as standard. Aliquots of each protein sample were either used immediately or stored at $-80{ }^{\circ} \mathrm{C}$.

Semi-quantitative RT-PCR. The entire semi-quantitative RT-PCR procedure, including the isolation of parasite total RNA from various parasite life-cycle stages, the normalization of parasite RNA contents in intracellular samples, reverse transcription, amplification and analysis, has been described in detail by Millership et al. (2004a, b). In this study, the following primer pair was used to amplify CpACBP1 transcripts: CpACBP1-F334 (5'-CCTTTATTAGAATCAAACCTGG-3') and CpACBP1-R334 (5'-TTGGATAGGAGTCAAACCATC-3'). Another pair of previously reported primers $(995 \mathrm{~F}$, 5'-TAGAGATTGGAGGTTGTTCCT-3' and 1206R, 5' -CTCCACCAACTAAGAACGGCC-3') was used to amplify $18 \mathrm{~S}$ rRNA as a control for normalization (Abrahamsen \& Schroeder, 1999). Each semiquantitative RT-PCR reaction contained a comparable amount of parasite RNA and was subjected to 45 min reverse transcription and 23 (for CpACBP1) or 20 (for $18 \mathrm{~S}$ rRNA) thermal cycles of PCR amplification. After agarose gel electrophoresis, the intensity of each product was measured using the GENETOOLS program v.3 (Hitachi Software Engineering) and the relative level of CpACBP1 transcripts was determined as the signal ratio between the CpACBP1 and rRNA amplicons.

Production of polyclonal antibodies to CpACBP. Polyclonal antibodies to recombinant CpACBP1 protein were raised in a pathogen-free rabbit. Initial immunization used $0.2 \mathrm{mg}$ affinity-purified MBP-CpACBP1 protein emulsified in an equal volume of complete Freund's adjuvant. Two subsequent booster immunizations $(0 \cdot 1 \mathrm{mg})$ were injected at 30 and 60 days, respectively, after the primary immunization. Rabbit sera were collected prior to and after the immunization protocol. The anti-MBP portion of the polyclonal antibodies was removed by absorbing antiserum with an equal volume of amylose-resin conjugated with MBP. The antibody titre and specificity were evaluated by Western blot analysis.

Western blot analysis. Western blot analysis was performed to test for the presence of CpACBP1 protein at various parasite lifecycle stages. Oocysts $\left(5 \times 10^{6}\right.$ per lane), free sporozoites $\left(2 \times 10^{7}\right.$ per lane) and HCT- 8 cells $\left(1 \times 10^{6}\right.$ per lane $)$ infected with $C$. parvum oocysts (oocysts/host cell ratio $=1: 1$ ) for 24,48 and $72 \mathrm{~h}$ were lysed in loading buffer containing a protease inhibitor cocktail for mammalian cells (Sigma-Aldrich) at $95^{\circ} \mathrm{C}$ for 8 min. After centrifugation for $5 \mathrm{~min}$, soluble materials were fractionated in a $10 \%$ SDS-PAGE gel and transferred onto a nitrocellulose membrane. The membrane was first blocked with $5 \%$ BSA in TBS $(20 \mathrm{mM}$ Tris, pH $7 \cdot 5$, $50 \mathrm{mM} \mathrm{NaCl}$ ) for $1 \mathrm{~h}$ and then incubated with rabbit anti-CpACBP 
antibodies and a monoclonal anti-rabbit IgG antibody conjugated to alkaline phosphatase in $1 \%$ BSA in TTBS (TBS with $0.05 \%$ Tween20). The blot was washed three times with TTBS after each incubation step and all procedures were performed at room temperature. Finally, the labelled proteins were developed using 5'-bromo-4chloro-3-indolyl phosphate (BCIP).

Immunofluorescence microscopy. Intracellular parasites were prepared by infecting HCT-8 cells grown on poly-L-lysine-treated glass coverslips for 24,48 or $72 \mathrm{~h}$. Cells were fixed with $10 \%$ formalin, rinsed with $\mathrm{PBS}$, extracted with cold methanol $\left(-20^{\circ} \mathrm{C}\right.$ for $5 \mathrm{~min}$ ), blocked in $0.5 \%$ BSA-PBS (10 min), labelled with primary antibodies ( $1 \mathrm{~h}$ in $0.5 \%$ BSA-PBS) and incubated with secondary antibodies conjugated with FITC or TRITC (60 min in $0.5 \%$ BSAPBS). Samples were washed after each incubation step (three times, 5 min each) using PBS. Free sporozoites were fixed in suspension, directly applied onto poly-L-lysine-treated coverslips, extracted and air-dried prior to incubation with antibodies. Co-localization of CpACBP1 with total membrane proteins (TMPs) and with a surfactin production element (SFP)-type phosphopantetheinyl transferase (CPSFP-PPT) immunolocalization was similarly performed, except that the respective rabbit antibodies were directly labelled with Alexa Fluor 488 or Alexa Fluor 546 using the appropriate fluorophorelabelling kits (Invitrogen) prior to the immunolabelling experiments. No secondary antibodies were used in co-localization experiments. The TMP antibody has been shown previously to mainly label the PVM and feeder organ in intracellular parasites (Chen et al., 2003), while cytosolic localization of CPSFP-PPT has also been reported previously (Cai et al., 2005). All samples were mounted using a SlowFade Light Antifade medium containing 4',6'-diamidino-2phenylindole (DAPI) for DNA counter-staining (Invitrogen) and examined with an Olympus BX51 Epi-Fluorescence microscope equipped with differential interference contrast and FITC/TRITC/ DAPI filters.

Acyl-CoA binding assay. The binding affinity of CpACBP1 with fatty acyl-CoA was measured by a Lipidex 1000 assay as previously described (Rasmussen et al., 1990; Rosendal et al., 1993). Briefly, 40 pmol recombinant CpACBP1 was mixed with $\left[{ }^{14} \mathrm{C}\right]$ palmitoylCoA $(0-8 \mu \mathrm{M})$ in $100 \mu \mathrm{l}$ binding buffer $(10 \mathrm{mM}$ potassium phosphate, $\mathrm{pH} \mathrm{7 \cdot 4)}$ and incubated at $37^{\circ} \mathrm{C}$ for $30 \mathrm{~min}$. The mixture was then chilled on ice for $10 \mathrm{~min}$, mixed with $0.6 \mathrm{ml}$ cold Lipidex 1000 (hydroxyalkoxypropyl dextran, Type VI; Sigma-Aldrich) (50\%, v/v, slurry in binding buffer) with gentle rotation at $4{ }^{\circ} \mathrm{C}$ for $1 \mathrm{~h}$ and centrifuged for $5 \mathrm{~min}$ at $12000 \mathrm{~g}$ at $4{ }^{\circ} \mathrm{C}$ to remove free acyl-CoA esters. An aliquot of $200 \mu \mathrm{l}$ supernatant was taken from each sample for counting radioactivity in a Beckman LS6500 scintillation counter. Each experiment included negative controls using MBP-tag only for background subtraction. At least three replicates were performed for each experimental condition. The dissociation constant $\left(K_{\mathrm{d}}\right)$ was determined by plotting the amount of bound substrate against the total concentration of substrate using non-linear regression and Prism v4.0 (GraphPad Software).
The substrate preference for CpACBP1 was determined by a competition-binding assay. It was performed in $100 \mu \mathrm{l}$ binding buffer containing 40 pmol recombinant CpACBP1 (or MBP in control groups) and $80 \mathrm{pmol}\left[{ }^{14} \mathrm{C}\right]$ palmitoyl-CoA in the absence or presence of 80 pmol non-radioactive fatty acyl-CoA esters of various chain lengths (ranging from 4 to 20 carbons). All samples were incubated, extracted with Lipidex 1000 and counted for radioactivity as described above.

In addition, we also tested whether CpACBP1 could specifically bind to long-chain fatty acids. In this assay, 80 pmol recombinant CpACBP1 protein was incubated with $80 \mathrm{pmol}\left[{ }^{3} \mathrm{H}\right]$ palmitic acid or $\left[{ }^{14} \mathrm{C}\right]$ palmitoyl-CoA in $100 \mu \mathrm{l}$ buffer. After extraction with Lipidex 1000 , the supernatants were counted for radioactivity as described above.

Autoradiography. To visualize the binding of CpACBP1 with acyl-CoA, 80 pmol fusion protein was incubated with 80 pmol $\left[{ }^{14} \mathrm{C}\right]$ palmitoyl-CoA in the presence or absence of 80 pmol nonradioactive palmitoyl-CoA in $100 \mu$ binding buffer for $30 \mathrm{~min}$ at $37^{\circ} \mathrm{C}$. Subsequently, $20 \mu \mathrm{l}$ of the reaction was mixed with $5 \mu \mathrm{l} 5 \times$ native loading buffer and fractionated in a $10 \%$ native PAGE gel. The gel was dried on a heated gel drier and radioactive protein bands were visualized with X-ray film in a BioMax TranScreen LE intensifying system (Kodak).

\section{RESULTS}

\section{CpACBP1 is a 'long-type' ACBP containing ankyrin repeats}

CPACBP1 is the only ACBP homologue that can be identified from the $C$. parvum genome by repeated BLAST searches using several animal, plant and protist ACBP proteins as queries. This intronless gene encodes 268 aa that constitute an N-terminal ACBP domain ( $\sim 90$ aa) and a C-terminal sequence $(\sim 178$ aa) containing two ankyrin repeats (Fig. 1). Other apicomplexans appear to possess more ACBP homologues in their genomes. For example, $P$. falciparum has four ACBP homologues (three short and one long), and T. gondii has two (one short and one long). The small ACBP domains in all apicomplexan proteins share many conserved residues with their homologues found in animals and plants that are characteristic to this group of proteins (Fig. 2). Amino acids that are critical to ligandbinding activity are all present in CpACBP1 and other apicomplexan ACBPs (Fig. 2, indicated by solid dots) (Burton et al., 2005). Similar to CpACBP1, the long-type ACBP in T. gondii contains two ankyrin repeats (Fig. 1). However, it also possesses an $\mathrm{N}$-terminal signal peptide, possibly for secretion. On the other hand, the P. falciparum

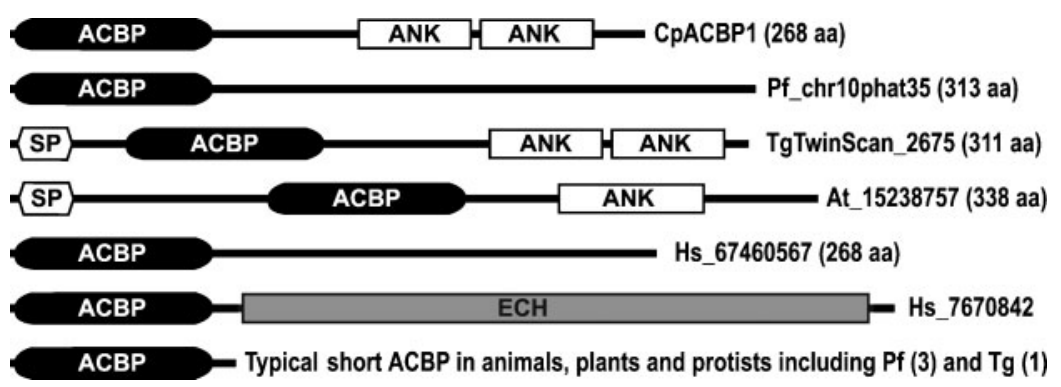

Fig. 1. Domain organization of CpACBP1 in comparison to those from other representative eukaryotic ACBPs. 


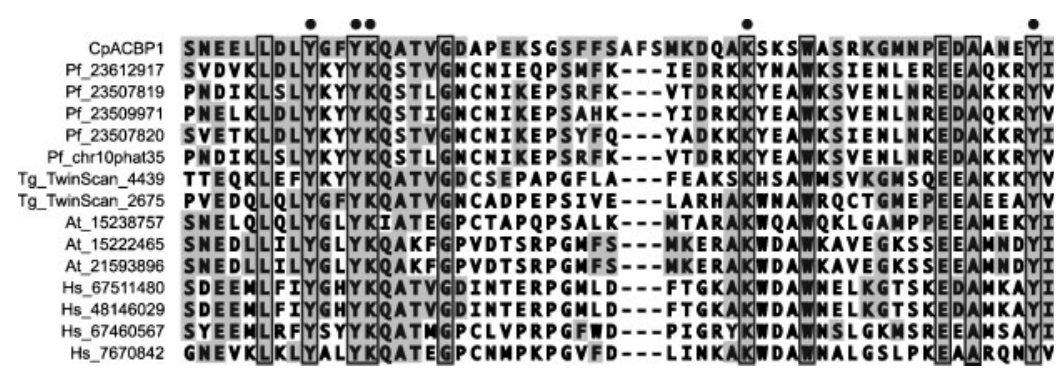

Fig. 2. Multiple alignment of the conserved region in the $\mathrm{ACBP}$ domain between CpACBP1 and other representative eukaryotic ACBPs. Amino acids shared between CpACBP1 and other sequences are shaded, while residues conserved among all listed sequences are boxed. Pf, Plasmodium falciparum; Tg, Toxoplasma gondii; At, Arabidopsis thaliana; Hs, Homo sapiens. Numbers following the species abbreviations are the accession numbers in GenBank or the corresponding apicomplexan genome databases (www.PlasmoDB.org or www. ToxoDB.org). Solid dots indicate amino acids critical to acyl-CoA-binding activity.

long-type ACBP contains no ankyrin repeats (Fig. 1). These observations indicate that apicomplexans may differ from each other by possessing different numbers and types of ACBPs.

\section{CpACBP1 has highest binding affinity to C16:0 palmitoyl-CoA}

To investigate the binding features of CpACBP1, we expressed the full-length protein as well as the ACBP domain as MBP-fusion proteins (MBP-CpACBP1 and MBP-CpACBP1-S). Both fusion proteins were purified to homogeneity using amylose-resin-based affinity chromatography (Fig. 3). Although the fusion proteins expressed using pMAL-c2 $\mathrm{x}$ vector contain a factor $\mathrm{X} \alpha$ cleavage site, attempts to effectively remove the MBP-tag using factor X $\alpha$ were not successful. It is possible that the MBP-CpACBP1 fusion protein folds in a way that interferes with the access of

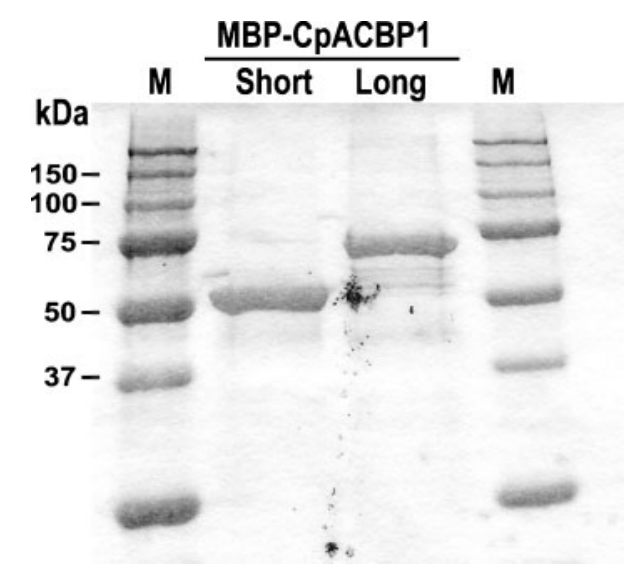

Fig. 3. SDS-PAGE analysis of purified MBP-fused CpACBP1 proteins. Short, MBP-fusion protein containing ACBP domain only; long, MBP-fusion protein containing the entire CpACBP1 sequence; $M$, protein molecular marker. factor $\mathrm{X} \alpha$ to the cleavage site. Difficulties in removing the MBP-tag have also been found in studies using recombinant C. parvum malate and lactate dehydrogenases in which their activities were assayed in the presence of MBP-tag (Madern et al., 2004).

Using the Lipidex 1000 assay, we first confirmed that the intact MBP-CpACBP1 was able to specifically bind to palmitoyl-CoA, while MBP had no or little affinity for the same ligand (Fig. 4a). With both ligand and protein concentrations at $80 \mu \mathrm{M}$ (near the highest concentration of $100 \mu \mathrm{M}$ in the kinetics assay), the non-specific binding of MBP-tag accounted for $\sim 5 \%$ of the specific binding observed for MBP-CpACBP1. In another assay using both palmitic acid and palmitoyl-CoA as substrates, both MBPtag and MBP-CpACBP1 displayed almost the same amount of radioactivity when binding to palmitic acid (Fig. 4b), indicating that fatty acids are not the favourite ligands for CpACBP1. Based on these observations, we decided to use uncleaved fusion proteins in all subsequent analyses.

We first studied the specific binding between CpACBP1 and palmitoyl-CoA, and determined that the $K_{\mathrm{d}}$ for CpACBP1 binding to palmitoyl-CoA was $407 \mathrm{nM}$ (Fig. 4c). This value is significantly higher than those reported for many other ACBPs which are typically in the $1-10 \mathrm{nM}$ range (Burton et al., 2005). This observation suggests that, although the Lipidex assay is a reliable assay for determining the acylCoA-binding profile for an ACBP, it is probably not a sensitive method for determining binding kinetics due to the binding competition between ACBP and Lipidex 1000 during the extraction step (Rasmussen et al., 1994). It is also possible that a significant portion of the CpACBP1 fusion protein was inactive and incapable of binding to acylCoA, and/or the N-terminal $42 \mathrm{kDa}$ MBP-tag might physically interfere with the binding kinetics of the recombinant proteins.

We also tested the specific binding of recombinant CpACBP1-S to palmitoyl-CoA in comparison to fulllength CPACBP1 using the same Lipidex assay. With 
(a)

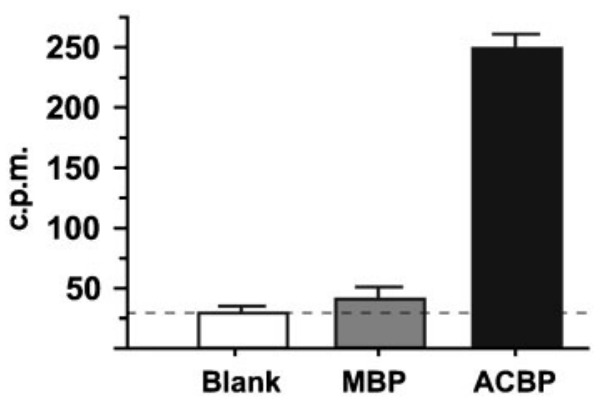

(b)

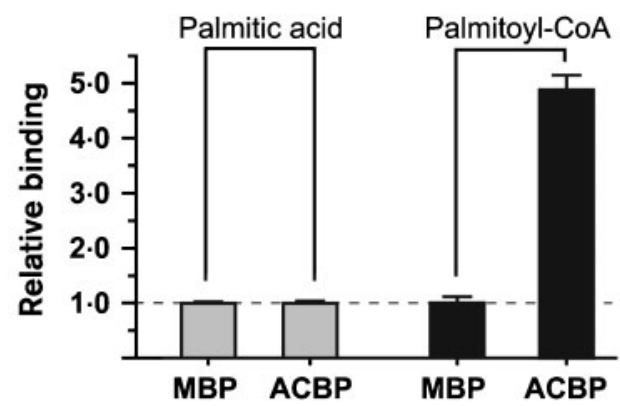

(c)

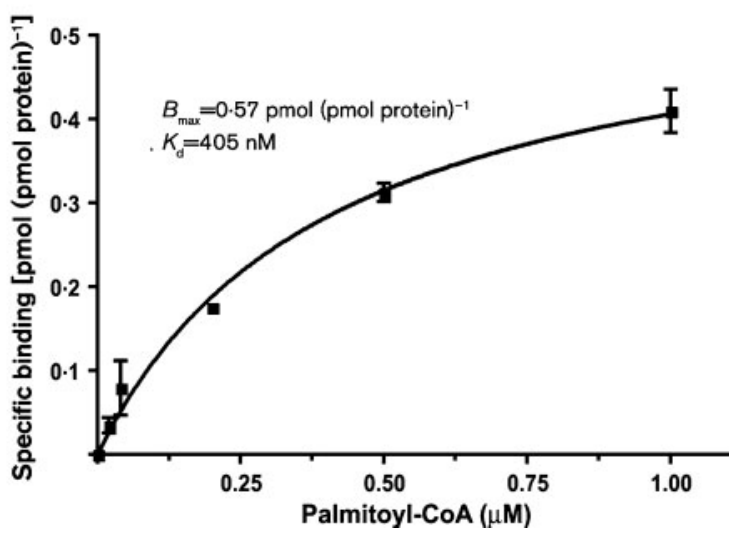

Fig. 4. (a) Specific and non-specific binding of $\left[{ }^{14} \mathrm{C}\right]$ palmitoylCoA $(80 \mu \mathrm{M})$ by MBP-fused CpACBP1 $(40 \mu \mathrm{M})$ and MBP-tag $(40 \mu \mathrm{M})$ as determined by the Lipidex 1000 extraction assay. (b) Relative binding between CpACBP1 and $\left[{ }^{14} \mathrm{C}\right]$ palmitoylCoA $(80 \mu \mathrm{M})$ or $\left[{ }^{3} \mathrm{H}\right]$ palmitic acid as determined by the Lipidex 1000 assay. Radioactivity was normalized using MBP-tag as control. (c) Binding kinetics of recombinant CpACBP1 with palmitoyl-CoA as determined by the Lipidex 1000 assay (see Methods).

$0 \cdot 4 \mu \mathrm{M}$ protein and $0 \cdot 8 \mu \mathrm{M}$ palmitoyl-CoA, both fusion proteins displayed similar specific-binding activities $\left[0 \cdot 180\right.$ and $0 \cdot 206 \mathrm{pmol}(\mathrm{pmol} \text { protein })^{-1}$ for CpACBP1 and CpACBP1-S, respectively], thus confirming that the ACBP domain was responsible for acyl-CoA binding.

The specific binding of CpACBP1 to fatty acyl-CoAs was further confirmed by autoradiography. When $\left[{ }^{14} \mathrm{C}\right]-$ palmitoyl-CoA was incubated with various fusion proteins,
CpACBP1

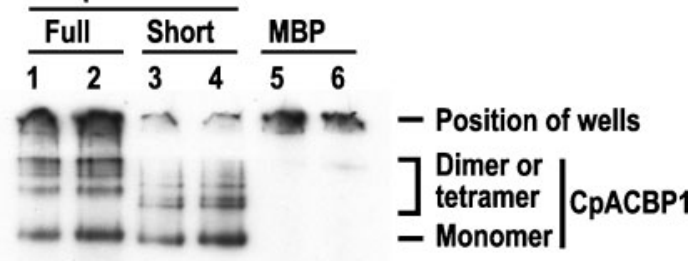

Fig. 5. Autoradiography showing the binding of $\left[{ }^{14} \mathrm{C}\right]$ palmitoylCoA by full-length (lanes 1 and 2) and short ACBP-domain only (lanes 3 and 4) fusion proteins after native PAGE fractionation. MBP-tag only (lanes 5 and 6) was used as control. In lanes 1,3 and 5 , the same molar amount of non-radioactive palmitoyl-CoA was also included to compete with the radioactive acyl-CoA, resulting in the reduced intensity of radioactivity in these lanes. Positions of CpACBP1 monomers, dimers and polymers are indicated. Some acyl-CoA molecules were retained in the loading wells as indicated.

only recombinant CpACBP1 (full-length) or CpACBP1-S (ACBP domain only), but not MBP-tag, displayed radioactivity (Fig. 5). The intensity of radioactivity associated with fractionated CpACBP1 was reduced when an equimolar amount of non-radioactive fatty acyl-CoA was added to the reaction. It was also observed that multiple radioactive bands appeared in lanes containing CpACBP1. Since protein fractionation was performed in native polyacrylamide gels, this observation suggests that CPACBP1 may also function as a dimer or tetramer. However, it is also possible that protein aggregation might occur under the experimental conditions used.

Based on the specific-binding data, we decided to test the substrate preference for CpACBP1 using $0 \cdot 8 \mu \mathrm{M}$ of various fatty acyl-CoAs in competition with equimolar concentrations of palmitoyl-CoA. This concentration is approximately twice that of the $K_{\mathrm{d}}$ value for palmitoyl-CoA, so that the protein occupancy by the ligands was neither too low nor too high. The results show that CpACBP1 can bind to medium- and long-chain acyl-CoAs (Fig. 6). This protein has highest affinity for palmitoyl-CoA (C16:0), but decreased binding affinity for other acyl-CoA esters. However, CpACBP1 is incapable of binding acyl-CoA esters with an acyl chain of 20 carbons or longer. This feature makes CpACBP1 different to many other ACBPs, including those from bovines and trypanosomes that can bind to acyl-CoA esters with an acyl chain of 24 or more carbons (Milne \& Ferguson, 2000; van Aalten et al., 2001). 


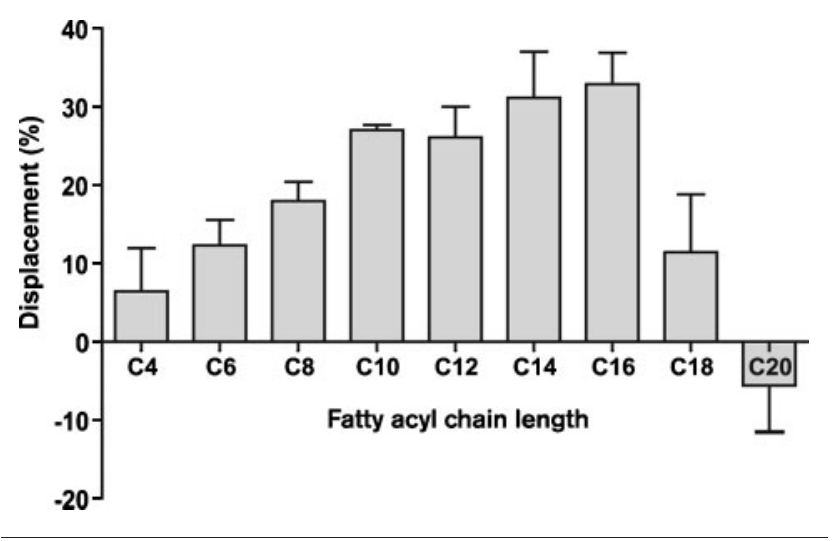

Fig. 6. Acyl-CoA binding specificity of $\mathrm{CpACBP} 1$ determined by the Lipidex 1000 competition binding assay. In each reaction, $\left[{ }^{14} \mathrm{C}\right]$-palmitoyl-CoA was mixed with an equimolar amount of non-radioactive acyl-CoA with specified carbon chain length. Binding affinity is presented as the percentage displacement of radioactive palmitoyl-CoA by non-radioactive acyl-CoA. SEM values were determined from at least three individual samples.

\section{CpACBP1 is differentially expressed and its encoded protein is probably localized to the PVM}

Semi-quantitative RT-PCR showed that CPACBP1 is expressed differentially in the complex parasite life cycle (Fig. 7). CPACBP1 transcripts were barely detectable in free sporozoites, but started to appear after parasite invasion into host cells. The level of $C P A C B P 1$ transcripts was relatively low during early intracellular development (from 3 to $24 \mathrm{~h}$ post-infection), but gradually increased with the time of infection. This expression pattern differs from that of many other C. parvum genes, such as the replication protein A subunits, CpSFP-PPT and $\beta$-tubulin, but is similar to those of oocyst wall proteins in this parasite (Abrahamsen \& Schroeder, 1999; Cai et al., 2005; Millership et al., 2004a;

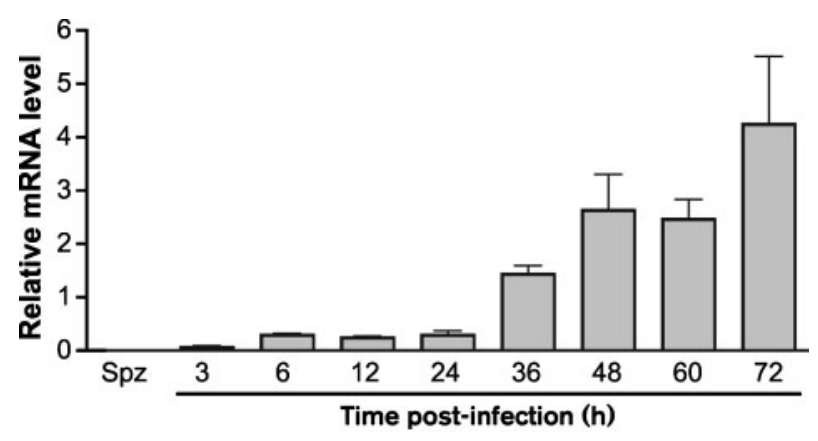

Fig. 7. Relative levels of CpACBP1 gene transcripts in various C. parvum life-cycle stages as determined by semi-quantitative RT-PCR. The level of transcripts is normalized using that of the parasite 18S rRNA as a control. Spz, Excysted free sporozoites.
Rider et al., 2005). Such a differential expression pattern is also supported by Western blot and immunofluorescence microscopic analyses that only detected CpACBP1 protein in the intracellular parasites, but not in oocysts or free sporozoites (Figs 8 and 9).

More surprisingly, immunofluorescence microscopy indicates that CpACBP1 is probably located on the PVM (Fig. 9). Rabbit polyclonal antibodies clearly labelled the surface of meronts with a homogeneous pattern of distribution, but failed to label the merozoites within the meronts (Fig. 9a). In a dual-labelling experiment using a rabbit polyclonal antibody mainly against PVM and the electron-dense connection between host cell and parasite, we co-localized CpACBP1 and PVM proteins (Fig. 9b). On the other hand, a polyclonal antibody against CpSFP-PPT clearly labelled the merozoites, but not on the surface of meronts in another dual-labelling experiment (Fig. 9c).

\section{DISCUSSION}

ACBP was originally identified as a mammalian diazepambinding inhibitor - a neuropeptide that could inhibit diazepam binding to the $\gamma$-aminobutyric acid receptor (Guidotti et al., 1983). Typical ACBPs are small ( $\sim 10 \mathrm{kDa})$ cytosolic proteins (Burton et al., 2005). However, there are a number of long-type ACBPs that are fused with ankyrin repeats, such as ACBP1 and ACBP2 in A. thaliana (Chye et al., 1999; Li \& Chye, 2003), or with other functional domains, such as the human peroxisomal D3, D2-enoyl-CoA isomerase (Geisbrecht et al., 1999). ACBP mainly functions as an intracellular acyl-CoA transporter and pool former, and is critical to lipid metabolism in cells (Gossett et al., 1996; Knudsen et al., 2000; Schroeder et al., 1998). However, ACBP has only been found in eukaryotes, not in prokaryotes except for a few pathogenic eubacteria that might have acquired ACBP from eukaryotic hosts via lateral gene transfer (Burton et al., 2005).

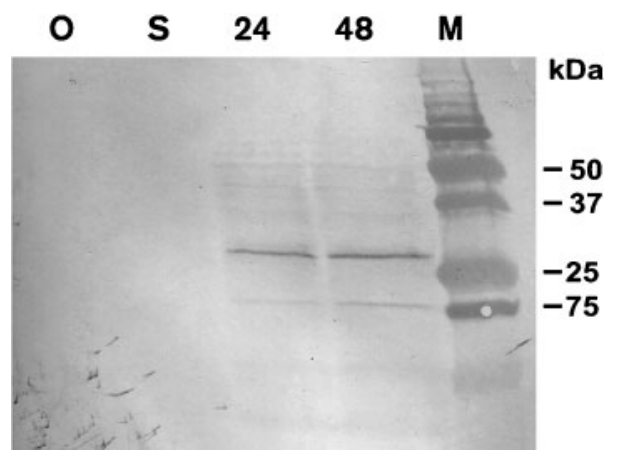

Fig. 8. Western blot detection of CpACBP1 protein in $C$. parvum oocysts (O), excysted free sporozoites (S) and intracellular parasites grown for 24 and $48 \mathrm{~h}$. CpACBP1 was only detected in the intracellular parasites, but not in oocysts and free sporozoites. 


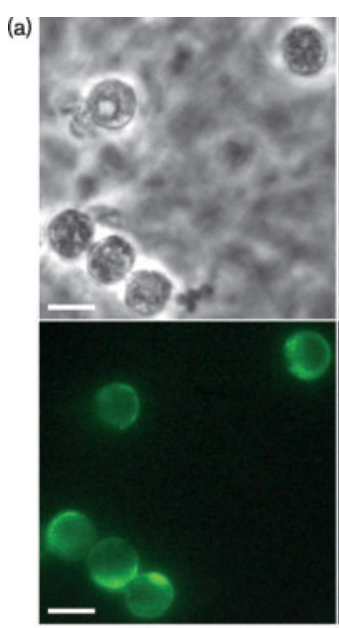

$24 \mathrm{~h}$

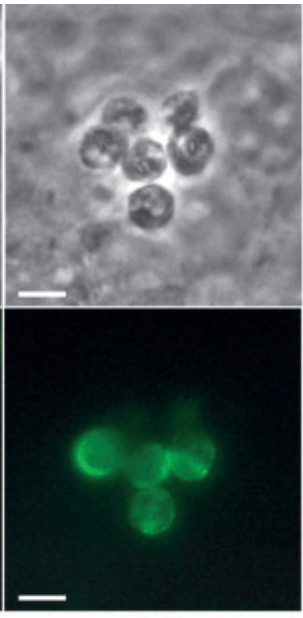

$48 \mathrm{~h}$

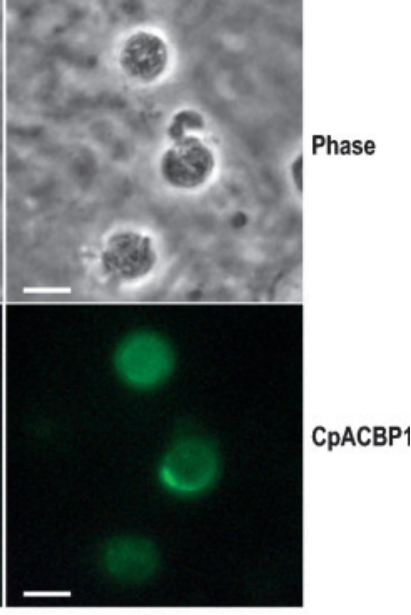

$72 \mathrm{~h}$

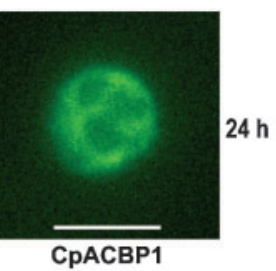

(c)

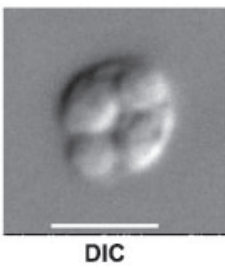

(b)

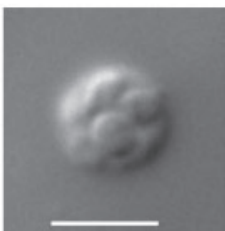

DIC

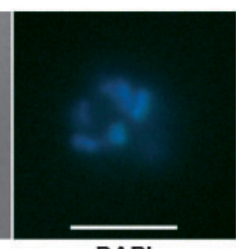

DAPI

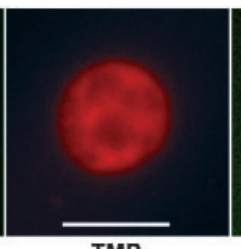

TMP
CpACBP1

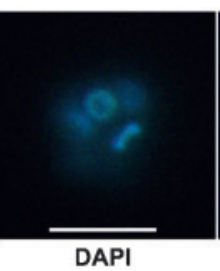

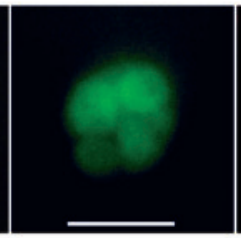

CpSFP-PPT

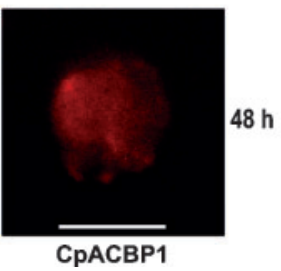

Fig. 9. Immunofluorescence microscopy of the CpACBP1 protein in intracellular $C$. parvum. (a) Indirect immunolabelling of intracellular parasites grown for 24,48 and $72 \mathrm{~h}$ using a rabbit polyclonal antibody against CpACBP1 and a secondary antibody conjugated with FITC. (b) Direct dual-labelling of intracellular parasites grown for $24 \mathrm{~h}$ using an Alexa Fluor 488-conjugated antibody against CpACBP1 and an Alexa Fluor 546conjugated antibody against parasite TMPs that mainly labels PVM and feeder organ. Both antibodies displayed the same labelling pattern on the surface of a meront. (c) Duallabelling of intracellular parasites grown for $48 \mathrm{~h}$ using an Alexa Fluor 546-conjugated antibody against CpACBP1 and an Alexa Fluor 488-conjugated antibody against cytosolic CpSFP-PPT, showing CpACBP1 mainly on the surface of meronts, rather than in merozoites. Phase, Phase-contrast; DIC, differential interference contrast; DAPI was used for counterstaining nuclei. Bars, $5 \mu \mathrm{m}$.
Among apicomplexans, C. parvum only possesses a single, long-type ACBP that is fused with an ankyrin repeats domain. However, other apicomplexans may have multiple ACBPs of various types (short-type, long-types fused with ankyrin repeats or with uncharacterized sequences) (Fig. 1), indicating that the ACBP-mediated metabolic pathways may be highly divergent in the Apicomplexa. Arabidopsis ACBP1 and ACBP2 are membrane proteins that differ from typical cytosolic ACBPs (Chye et al., 1999; Leung et al., 2005). The ankyrin repeats in these proteins are responsible for docking these proteins to the membrane by interacting with an ethylene-responsive element-binding protein. Our immunolabelling data indicate that CpACBP1 is also a membrane protein (i.e. mainly associated with PVM) (Fig. 9). Such a membrane association is probably mediated by the interaction of ankyrin repeats with a yet unknown protein(s) in the PVM.

Although C. parvum is an intracellular parasite, it does not reside within the host cytoplasm. Instead, this parasite is extracytoplasmic, covered by PVM on the surface of intestinal epithelial cells (Chen et al., 2002). Therefore, the PVM is the only barrier separating parasites from the intestinal lumen. The localization of CpACBP1 to the PVM is thus intriguing, although it is currently uncertain whether
CpACBP1 is also associated with the feeder organ at the host cell-parasite interface. It implies that CpACBP1 may be involved in the formation of the PVM or uptake of fatty acids across the PVM. However, since CPACBP1 mRNA and protein are undetectable (or barely detectable) in sporozoites and in the invasion stages (i.e. the first $3 \mathrm{~h}$ of infection), it seems less likely that CpACBP1 is associated with the early stage of PVM formation. On the other hand, it is known that $C$. parvum may have to import fatty acids from host cells or the intestinal lumen since it is likely to be incapable of synthesizing fatty acids de novo, although it is capable of elongating long-chain fatty acids (Zhu, 2004). Therefore, it is possible that CpACBP1 may function as a fatty acyl-CoA scavenger in conjunction with an acyl-CoA synthetase on or around the PVM (or the feeder organ) to facilitate fatty acid uptake by the parasite.

Another possibility is that CpACBP1 may be involved in the synthesis of GPI anchor. In African trypanosomes, ACBP has been found to be responsible for supplying myristoyl-CoA to the fatty acid remodelling machinery during GPI synthesis (Milne \& Ferguson, 2000; Milne et al., 2001). Although it is as yet unclear whether GPI-anchored molecules are present in the PVM, a recent comprehensive chemical analysis has clearly revealed the presence 
of complex glycosylinositol phospholipids in C. parvum sporozoites (Priest et al., 2003). In addition, a number of enzymes involved in the biosynthesis of GPI anchors are also present in the C. parvum genome, including phosphatidylinositol $N$-acetylglucosaminyltransferases (e.g. GenBank accession numbers XP_628152, XP_627129 and XP_626317). Nonetheless, further investigations are necessary to test these hypotheses.

Our biochemical data show that, although CpACBP1 can bind to medium- to long-chain fatty acids with chain lengths up to 18 carbons, it displays the highest binding affinity towards to the C16:0 palmitoyl-CoA. The $K_{d}$ value of $407 \mathrm{nM}$ obtained by the Lipidex 1000 assay is comparable to those of other ACBPs determined by the same assay (Rasmussen et al., 1994). However, this value does not represent the true acyl-CoA binding affinity. Rather, it reflects the competitive binding between ACBP and Lipidex 1000 (Rasmussen et al., 1994). The $K_{\mathrm{d}}$ values determined by fluorescence or dialyser-based methods are typically lower, in the range of 1-10 nM (Chao et al., 2002; Frolov \& Schroeder, 1998; Milne \& Ferguson, 2000; van Aalten et al., 2001; Wadum et al., 2002). On the other hand, although Lipidex 1000 cannot be used to assess the true binding affinity of ACBPs, this method can be used as a qualitative assessment, such as the ligand competition assay. Autoradiography indicates that both the full-length CpACBP1 protein and its ACBP domain may form dimers or even polymers (Fig. 4c). However, it is unclear whether CpACBP1 is truly present as a dimer or polymers in vivo, or whether the observed multiple bands in autoradiography are only artefacts induced by the experimental conditions.

Fatty acids are essential to all organisms. Recently, fatty acid metabolism has been considered as a promising target for drug development against cryptosporidiosis and other important pathogenic apicomplexans (Gornicki, 2003; Kuo et al., 2003; Ralph et al., 2001; Roberts et al., 2003; Waller et al., 2003; Zhu, 2004). Because ACBP plays a critical role in fatty acid metabolism, it is reasonable to speculate that CpACBP1 and other apicomplexan ACBPs may be explored as new drug targets for the control of cryptosporidiosis or other apicomplexan-based diseases.

\section{ACKNOWLEDGEMENTS}

We thank Jason Millership and Palvi Waghela for technical assistance. This research was supported by a grant from National Institutes of Health (R01 AI44594).

\section{REFERENCES}

Abrahamsen, M. S. \& Schroeder, A. A. (1999). Characterization of intracellular Cryptosporidium parvum gene expression. Mol Biochem Parasitol 104, 141-146.
Abrahamsen, M. S., Templeton, T. J., Enomoto, S. \& 17 other authors (2004). Complete genome sequence of the apicomplexan, Cryptosporidium parvum. Science 304, 441-445.

Bradford, M. M. (1976). A rapid and sensitive method for the quantitation of microgram quantities of protein utilizing the principle of protein-dye binding. Anal Biochem 72, 248-254.

Burton, M., Rose, T. M., Faergeman, N. J. \& Knudsen, J. (2005). Evolution of the acyl-CoA binding protein (ACBP). Biochem J 392, 299-307.

Cai, X., Herschap, D. \& Zhu, G. (2005). Functional characterization of an evolutionarily distinct phosphopantetheinyl transferase in the apicomplexan Cryptosporidium parvum. Eukaryot Cell 4, 1211-1220.

Chao, H., Martin, G. G., Russell, W. K., Waghela, S. D., Russell, D. H., Schroeder, F. \& Kier, A. B. (2002). Membrane charge and curvature determine interaction with acyl-CoA binding protein (ACBP) and fatty acyl-CoA targeting. Biochemistry 41, 10540-10553.

Chappell, C. L. \& Okhuysen, P. C. (2002). Cryptosporidiosis. Curr Opin Infect Dis 15, 523-527.

Chen, X. M., Keithly, J. S., Paya, C. V. \& LaRusso, N. F. (2002). Cryptosporidiosis. N Engl J Med 346, 1723-1731.

Chen, X. M., Huang, B. Q., Splinter, P. L., Cao, H., Zhu, G., McNiven, M. A. \& LaRusso, N. F. (2003). Cryptosporidium parvum invasion of biliary epithelia requires host cell tyrosine phosphorylation of cortactin via c-Src. Gastroenterology 125, 216-228.

Chye, M. L., Huang, B. Q. \& Zee, S. Y. (1999). Isolation of a gene encoding Arabidopsis membrane-associated acyl-CoA binding protein and immunolocalization of its gene product. Plant $J \mathbf{1 8}$, 205-214.

Frolov, A. \& Schroeder, F. (1998). Acyl coenzyme A binding protein. Conformational sensitivity to long chain fatty acyl-CoA. J Biol Chem 273, 11049-11055.

Geisbrecht, B. V., Zhang, D., Schulz, H. \& Gould, S. J. (1999). Characterization of PECI, a novel monofunctional Delta(3), Delta(2)enoyl-CoA isomerase of mammalian peroxisomes. J Biol Chem 274, 21797-21803.

Gornicki, P. (2003). Apicoplast fatty acid biosynthesis as a target for medical intervention in apicomplexan parasites. Int $J$ Parasitol 33, 885-896.

Gossett, R. E., Frolov, A. A., Roths, J. B., Behnke, W. D., Kier, A. B. \& Schroeder, F. (1996). Acyl-CoA binding proteins: multiplicity and function. Lipids 31, 895-918.

Guidotti, A., Forchetti, C. M., Corda, M. G., Konkel, D., Bennett, C. D. \& Costa, E. (1983). Isolation, characterization, and purification to homogeneity of an endogenous polypeptide with agonistic action on benzodiazepine receptors. Proc Natl Acad Sci U S A 80, 3531-3535.

Knudsen, J., Neergaard, T. B., Gaigg, B., Jensen, M. V. \& Hansen, J. K. (2000). Role of acyl-CoA binding protein in acyl-CoA metabolism and acyl-CoA-mediated cell signaling. J Nutr 130, 294S-298S.

Kuo, M. R., Morbidoni, H. R., Alland, D. \& 14 other authors (2003). Targeting tuberculosis and malaria through inhibition of enoyl reductase: compound activity and structural data. J Biol Chem 278, 20851-20859.

Leung, K. C., Li, H. Y., Xiao, S., Tse, M. H. \& Chye, M. L. (2005). Arabidopsis ACBP3 is an extracellularly targeted acyl-CoA-binding protein. Planta, $1-11$.

Li, H. Y. \& Chye, M. L. (2003). Membrane localization of Arabidopsis acyl-CoA binding protein ACBP2. Plant Mol Biol 51, 483-492.

Madern, D., Cai, X., Abrahamsen, M. S. \& Zhu, G. (2004). Evolution of Cryptosporidium parvum lactate dehydrogenase from malate dehydrogenase by a very recent event of gene duplication. Mol Biol Evol 21, 489-497. 
Millership, J. J., Cai, X. \& Zhu, G. (2004a). Functional characterization of replication protein A2 (RPA2) from Cryptosporidium parvum. Microbiology 150, 1197-1205.

Millership, J. J., Waghela, P., Cai, X., Cockerham, A. \& Zhu, G. (2004b). Differential expression and interaction of transcription coactivator MBF1 with TATA-binding protein (TBP) in the apicomplexan Cryptosporidium parvum. Microbiology 150, 1207-1213.

Milne, K. G. \& Ferguson, M. A. (2000). Cloning, expression, and characterization of the acyl-CoA-binding protein in African trypanosomes. J Biol Chem 275, 12503-12508.

Milne, K. G., Guther, M. L. \& Ferguson, M. A. (2001). Acyl-CoA binding protein is essential in bloodstream form Trypanosoma brucei. Mol Biochem Parasitol 112, 301-304.

Priest, J. W., Mehlert, A., Arrowood, M. J., Riggs, M. W. \& Ferguson, M. A. (2003). Characterization of a low molecular weight glycolipid antigen from Cryptosporidium parvum. J Biol Chem 278, 52212-52222.

Ralph, S. A., D'Ombrain, M. C. \& McFadden, G. I. (2001). The apicoplast as an antimalarial drug target. Drug Resist Updat 4, 145-151.

Rasmussen, J. T., Borchers, T. \& Knudsen, J. (1990). Comparison of the binding affinities of acyl-CoA-binding protein and fattyacid-binding protein for long-chain acyl-CoA esters. Biochem J 265, 849-855.

Rasmussen, J. T., Faergeman, N. J., Kristiansen, K. \& Knudsen, J. (1994). Acyl-CoA-binding protein (ACBP) can mediate intermembrane acyl-CoA transport and donate acyl-CoA for beta-oxidation and glycerolipid synthesis. Biochem J 299, 165-170.

Rider, S. D., Jr, Cai, X., Sullivan, W. J., Jr, Smith, A. T., Radke, J., White, M. \& Zhu, G. (2005). The protozoan parasite Cryptosporidium parvum possesses two functionally and evolutionarily divergent replication protein A large subunits. J Biol Chem 280, 31460-31469.

Roberts, C. W., McLeod, R., Rice, D. W., Ginger, M., Chance, M. L. \& Goad, L. J. (2003). Fatty acid and sterol metabolism: potential antimicrobial targets in apicomplexan and trypanosomatid parasitic protozoa. Mol Biochem Parasitol 126, 129-142.

Roos, D. S., Crawford, M. J., Donald, R. G., Fraunholz, M., Harb, O. S., He, C. Y., Kissinger, J. C., Shaw, M. K. \& Striepen, B. (2002). Mining the Plasmodium genome database to define organellar function: what does the apicoplast do? Philos Trans R Soc Lond B Biol Sci $357,35-46$.
Rosendal, J., Ertbjerg, P. \& Knudsen, J. (1993). Characterization of ligand binding to acyl-CoA-binding protein. Biochem J 290, 321-326.

Schroeder, F., Jolly, C. A., Cho, T. H. \& Frolov, A. (1998). Fatty acid binding protein isoforms: structure and function. Chem Phys Lipids 92, $1-25$.

Thompson, R. C., Olson, M. E., Zhu, G., Enomoto, S., Abrahamsen, M. S. \& Hijjawi, N. S. (2005). Cryptosporidium and cryptosporidiosis. Adv Parasitol 59, 77-158.

Tzipori, S. \& Widmer, G. (2000). The biology of Cryptosporidium. Contrib Microbiol 6, 1-32.

van Aalten, D. M., Milne, K. G., Zou, J. Y., Kleywegt, G. J., Bergfors, T., Ferguson, M. A., Knudsen, J. \& Jones, T. A. (2001). Binding site differences revealed by crystal structures of Plasmodium falciparum and bovine acyl-CoA binding protein. J Mol Biol 309, 181-192.

Wadum, M. C., Villadsen, J. K., Feddersen, S., Moller, R. S., Neergaard, T. B., Kragelund, B. B., Hojrup, P., Faergeman, N. J. \& Knudsen, J. (2002). Fluorescently labelled bovine acyl-CoA-binding protein acting as an acyl-CoA sensor: interaction with CoA and acylCoA esters and its use in measuring free acyl-CoA esters and nonesterified fatty acids. Biochem J 365, 165-172.

Waller, R. F., Ralph, S. A., Reed, M. B., Su, V., Douglas, J. D., Minnikin, D. E., Cowman, A. F., Besra, G. S. \& McFadden, G. I. (2003). A type II pathway for fatty acid biosynthesis presents drug targets in Plasmodium falciparum. Antimicrob Agents Chemother 47, 297-301.

Zhu, G. (2004). Current progress in the fatty acid metabolism in Cryptosporidium parvum. J Eukaryot Microbiol 51, 381-388.

Zhu, G., Keithly, J. S. \& Philippe, H. (2000a). What is the phylogenetic position of Cryptosporidium? Int J Syst Evol Microbiol 50, 1673-1681.

Zhu, G., Marchewka, M. J., Woods, K. M., Upton, S. J. \& Keithly, J. S. (2000b). Molecular analysis of a Type I fatty acid synthase in Cryptosporidium parvum. Mol Biochem Parasitol 105, 253-260.

Zhu, G., LaGier, M. J., Stejskal, F., Millership, J. J., Cai, X. \& Keithly, J. S. (2002). Cryptosporidium parvum: the first protist known to encode a putative polyketide synthase. Gene 298, 79-89.

Zhu, G., Li, Y., Cai, X., Millership, J. J., Marchewka, M. J. \& Keithly, J. S. (2004). Expression and functional characterization of a giant Type I fatty acid synthase (CpFAS1) gene from Cryptosporidium parvum. Mol Biochem Parasitol 134, 127-135. 